\title{
Prevalence and outcome of Preterm Premature Rupture of Membranes (PPROM) among pregnant women attending Ain Shams maternity hospital
}

\author{
Hasnaa A. Abouseif ${ }^{1}$, Ayat F. Mansour ${ }^{1}$, Sheriene F. Hassan ${ }^{1}$ and Sahar M. Sabbour $^{1}$ \\ ${ }^{1}$ Department of Community, Environmental, and Occupational Medicine, Faculty of \\ Medicine, Ain Shams University
}

Received: June, 2017 Accepted: March, 2017

\begin{abstract}
:
Background: Preterm premature rupture of membranes (PPROM) is one of the leading identifiable causes of premature birth and it accounts for high rates of perinatal deaths in developed and developing countries. Objectives: The study was conducted to measure the prevalence of PPROM among pregnant women attending Ain Shams maternity hospital, and to identify the fetal/neonatal outcome among pregnant women presented with PPROM and potential factors associated with poor fetal/neonatal outcome. Method: A cross sectional study was conducted on two phases using data retrieved from computerized hospital information system (HIS), Ain Shams Maternity Hospital. Data about all deliveries from 2011-2015 was revised from Hospital Information System to calculate the frequency of PPROM during these years. A sample of 300 medical files of pregnant women who were diagnosed with PPROM before 37 weeks and admitted to the hospital in the year 2015 were then extracted from the hospital archive and revised using a checklist developed by the investigators. Relevant data was obtained from these medical files. Results: Prevalence of PPROM ranged from 2.4\% in 2011 to $4.7 \%$ in 2015 with the highest rate during 2013 (5.3\%). Only 4.3\% of women presented with PPROM developed chorio-amnionitis. Regarding fetal outcome, $61.3 \%$ of infants developed a poor fetal outcome including; (fetal death and ICU admission), while 38.7\% of infants had good fetal outcome (alive \& well). By multivariate analysis women employment (OR 1.9 CI 1.2 - 3.5), gush type of PPROM (OR 1.9 CI 1.4 - 3.6), history of chorioamnionitis (OR 14.5 CI 12.4 - 132.1), delivery by CS (OR 2.5 CI $2.01-4.5$ ) and later gestational age at PPROM (OR 0.8 CI 0.2 - 0.9) were significant independent predictors of poor fetal outcome. Conclusion \& Recommendations: Prevalence PPROM at Ain Shams maternity hospital is increasing during study duration with fetal/neonatal complications that necessitate proper antenatal care and proper management.
\end{abstract}

Key words: PPROM, fetal outcome, Ain Shams, maternity hospital.

Corresponding Author: Hasnaa A. Abou Seif

\section{Introduction}

Premature rupture of membranes (PROM) is the rupture of the fetal membranes before the onset of labor, if occurred before 37 weeks, it's called; the preterm premature rupture of fetal membranes (PPROM). ${ }^{1}$ There is variation in the prevalence of PPROM and this is due to the difference in the studied populations. Premature rupture of membranes occurs between 5 and $15 \%$ of all pregnancies. ${ }^{2}$ It was $1.8 \%$ in Oman and $8 \%$ in Bangladesh. While in Pakistan a prevalence of $9.6 \%$ was reported. ${ }^{3}$

The pathophysiology is complex and multifactorial. ${ }^{4}$ The most common cause of PPROM is idiopathic. ${ }^{5}$ The primary 
complication for the mother is the risk of infection which can lead to chorioamnionitis which is as high as 25$35 \%$, metritis after delivery and increases the need for CS delivery [6]. Complications of PPROM for the fetus and newborn consist of prematurity, cord compression which leads to fetal distress, neonatal sepsis, respiratory distress syndrome (RDS), and cord prolapse during rupture of membranes, placental abruption and risk of fetal and neonatal death. ${ }^{7}$

The fetal and neonatal morbidity and mortality risks are significantly affected by severity of oligo-hydramnious, duration of latency period and gestational age at PPROM. ${ }^{8}$

PPROM is one of the leading identifiable causes of premature birth and it accounts for approximately $18 \%$ to $20 \%$ of perinatal deaths in the United States [9], $30-40 \%$ of preterm deliveries in Oman and Iran. ${ }^{10,11}$

In Egypt, approximately $30 \%$ of preterm births are related to preterm rupture of membranes. $^{12}$ PPROM is a major complication of pregnancies and an important cause of perinatal morbidity and mortality. Currently, there is no effective way of preventing spontaneous rupture of fetal membranes due to ignorance of its etiology, with consequent inability to control its incidence. However, it is important that women be well informed regarding maternal, fetal and neonatal complications regardless of controversies of its management. ${ }^{13}$

So PPROM is critically important to be studied and to determine its impact as a clinical and public health problem. There is paucity of information regarding prevalence of PPROM and its outcome in Ain Shams maternity hospital so the aim of current study was to determine the extent of this problem (PPROM) and its complications.

Objectives: To measure the prevalence of PPROM among pregnant women attending Ain Shams maternity hospital during the years (2011 -2015) and to identify the outcome and the potential associated factors with poor fetal/neonatal outcome among pregnant women presented with PPROM.

\section{Methods}

Type, place \& population of the study: This study was conducted as a cross sectional study on two phases using data retrieved from the computerized hospital information system (HIS), in Ain Shams Maternity Hospital. Time of data collection: This Study lasted one year from January 2016 to December 2016. First phase included data collection about all deliveries from 2011-2015 obtained from the HIS. The second phase included retrieving a sample of files of pregnant women in 2015 who were diagnosed with PPROM before 37 weeks and admitted to Ain Shams maternity hospital. Diagnostic criteria of cases: Diagnosis of cases with PPROM was confirmed by obstetrics and gynecology physicians by presence of history of fluid drainage through the vagina, direct visualization of fluid from cervical os and ultrasound was done for fluid index. ${ }^{14}$ Sample size: To achieve the first study objective (prevalence of PPROM) total number of deliveries and total number of pregnant women who suffered from PPROM in each year from (20112015) were identified and included in calculating the prevalence in each year using the following equation:

Prevalence of PPROM= Total number of women presenting with PPROM/ Total number of deliveries in the same year and place. ${ }^{15}$

To achieve the second objective, sample size was calculated based on: frequency of expected poor neonatal outcome of PPROM from a previous study ; Mohamad $2013^{16}, \alpha$ error $=0.05$, Power $=0.8$ and accordingly, the sample size was calculated to be (300) patient 
Table (1): Prevalence of PPROM among women delivered at Ain Shams maternity hospital from 2011 to 2015.

\begin{tabular}{|c|c|c|c|c|c|}
\hline \multirow{2}{*}{ Year } & \multirow{2}{*}{$\begin{array}{l}\text { Number of } \\
\text { deliveries }\end{array}$} & \multirow{2}{*}{$\begin{array}{c}\text { Number of pregnant } \\
\text { women presenting with } \\
\text { PPROM } \\
\end{array}$} & \multirow{2}{*}{$\begin{array}{l}\text { Prevalence of } \\
\text { PPROM }\end{array}$} & \multicolumn{2}{|c|}{$95 \% \mathrm{CI}$} \\
\hline & & & & Lower & Upper \\
\hline 2011 & 13501 & 327 & $2.4 \%$ & 2.1 & 2.7 \\
\hline 2012 & 13966 & 446 & $3.1 \%$ & 2.8 & 3.4 \\
\hline 2013 & 13498 & 723 & $5.3 \%$ & 4.9 & 5.7 \\
\hline 2014 & 12868 & 634 & $5 \%$ & 4.6 & 5.4 \\
\hline 2015 & 12743 & 605 & $4.7 \%$ & 4.3 & 5.1 \\
\hline Total & 66576 & 2735 & $4.1 \%$ & 3.9 & 4.3 \\
\hline
\end{tabular}

files presented with PPROM using Power \&Sample size program.

In paper based archives, files were sorted in monthly piles. Files of females with PPROM were selected from year 2015 files until predetermined sample size (300 files) was satisfied. Patients' files of 2015 were the ones chosen because files of that year were the most recent and data is expected to be more or less complete.

Study tool: A checklist was developed by the investigators that included relevant variables from literature search $[15,17,18]$ and modified based on available data recorded in archived patient files. It contained the following items: Socio-demographic data (maternal age, occupation and smoking status). Obstetric data: (gravidity, gestational age at PPROM in weeks, previous history of PPROM, gestational age at delivery in weeks, type of pregnancy (single, twins, triple...), type of PPROM, latency period and history of chronic diseases of pregnant women. Maternal outcomes: Mode of delivery (spontaneous vaginal or CS delivery), presence of clinical chorioamnionitis which is characterized by maternal fever $\left(>39^{\circ} \mathrm{c}\right)$ accompanied by at least two of the following signs: maternal or fetal tachycardia, maternal leukocytosis, uterine tenderness, or foulsmelling amniotic fluid [19]. Fetal/Neonatal outcome: Birth weight (in $\mathrm{Kgs}$ ), alive and well, alive but needed Neonatal intensive care unit (NICU) admission, neonatal death or others (eg., neonatal infection-jaundice).

Pilot study: The checklist was tested for clarity and suitability for the aims of the study and to check for completeness and availability of patient files. Twenty patient files were extracted and completed in checklists. These files were paper based files from the archives of Ain Shams maternity hospital yet were not included in study sample.

Data management: Data were coded, entered and analyzed using SPSS program version 20. Operational definitions of variables: Gravidity was categorized into 3 groups: either primigravida (pregnant for the first time), multi gravida (pregnant $\geq$ twice) or grand multi gravida (pregnant $\geq 5$ times). ${ }^{3}$ Birth weight was classified into 2 groups: either low birth weight $\leq 2.5 \mathrm{Kg}$ or Normal birth weight $>2.5 \mathrm{Kg} .{ }^{20}$ Infant viability was classified into 3 groups: alive and well, alive but ill (including NICU admission, preterm birth, respiratory distress or infection) and neonatal death. Neonatal outcome was classified into 2 groups according to prognosis; either Poor neonatal outcome (occurrence of one or more of the following (NICU admission, preterm birth, respiratory distress or neonatal death) or Good neonatal outcome (born alive and well). ${ }^{18}$

Data Analysis: Quantitative variables were described as range and mean \pm SD. Qualitative variables were described as number and percentage with $95 \%$ CI of 


\section{Discussion:}

In the current study the prevalence of PPROM was $4.1 \%$ which agrees with a study conducted in India ${ }^{22}$, but it was lower than that reported in Bangladesh ${ }^{23}$ and high if compared to rates in Nigeria $^{24}$, Oman ${ }^{25}$ and in USA ${ }^{26}$. Higher rates of PPROM in the current study could be explained by the fact that Ain Shams Maternity hospital is a tertiary care level referral hospital to which complicated cases are usually referred. Also, cultural

Table (3): Fetal outcome of PPROM among neonates of studied women

\begin{tabular}{|c|c|c|}
\hline $\begin{array}{l}\text { Fetal outcome } \\
\text { of PPROM }\end{array}$ & $\begin{array}{c}\mathbf{N} \\
\mathbf{N}=300\end{array}$ & $\%$ \\
\hline \multicolumn{3}{|l|}{ Neonatal viability: } \\
\hline - Alive & 257 & 85.7 \\
\hline & 43 & \\
\hline \multicolumn{3}{|l|}{ Fetal outcome: } \\
\hline 1-Alive and well & 116 & 38.7 \\
\hline 2-A & 141 & 47.0 \\
\hline - NIC & 21 & 7.0 \\
\hline - $\mathrm{PT} \& \mathrm{RDS}{ }^{1}$ & 114 & 38.0 \\
\hline - Others (2) & 6 & 2.0 \\
\hline 3-Died & 43 & 14.3 \\
\hline \multicolumn{3}{|l|}{ Fetal outcome*: } \\
\hline - Good fetal outcome 3 & 116 & 38.7 \\
\hline - Poor fetal outco & 184 & 613 \\
\hline \multicolumn{3}{|l|}{ Infant Birth weight } \\
\hline & 62 & 20.7 \\
\hline - Low bi & 238 & \\
\hline \multicolumn{3}{|c|}{ Birth weight of infant at birth $(\mathrm{kg})$} \\
\hline Range (Min-Max) & $1-3.7$ & \\
\hline Mean \pm SD & $2 \pm 6$ & \\
\hline \multicolumn{3}{|c|}{$\begin{array}{l}\text { (1) PT = preterm \& RDS: respiratory distress } \\
\text { syndrome, (2) fetal infection-jaundice, (3) Good } \\
\text { outcome: born alive and well, (4) Poor } \\
\text { outcome: as neonatal NICU admission, } \\
\text { preterm birth, respiratory distress or } \\
\text { neonatal death }\end{array}$} \\
\hline
\end{tabular}

influences of early marriage, poverty resulting in low maternal weight gain and lack of birth spacing which are significantly associated with PPROM and this explanation was supported by Tahir et al. ${ }^{27}$ Moreover, inadequate antenatal care had a higher risk of poor maternal outcome according to a study done in Ismailia General Hospital [28]. Although there is a high utilization rate of antenatal care in Egypt (82\%) according to ${ }^{29}$ EDHS, 2016, the visits may be ineffective or inadequate.

In the present study the prevalence rate of PPROM among registered deliveries showed some fluctuations and rising frequency between 2011 till 2015. PPROM is considered to be increasing despite the minimal decline in 2014. A definite cause for this condition is still not clear however some factors which are already increasing in our society may have a role e.g. active and passive smoking.

Rate of chorioamnionitis in the current study was much lower than that reported in other countries ranged between $13 \%$ and $60 \%$ in Victoria, Ireland and in Bangladesh. ${ }^{30,15,31}$ This difference could be explained by the fact that most deliveries in the current study occurred within 24 hours after PPROM that could decrease the incidence of chorioamnionitis. However, lower rates of chorioamnionitis were reported in Iran and Zambia. ${ }^{32,18}$

Poor fetal outcome in the current study $(61.3 \%)$ was high compared to other studies $26.8 \%$ \& $34 \%$ in Zambia \& Ethiopia. ${ }^{18,20}$ One of the expected reasons may be the admission of late or complicated cases of PPROM that are probably referred to a tertiary care level as Ain Shams maternity hospital for better management so the chance of complications may be higher.

Fetal death occurred in $14.3 \%$, which is high if compared to 3.1 to $10 \%$ in other countries. ${ }^{18,22,32}$ The higher percentage of fetal death in the current study may be attributed to high rate of low birth weight (LBW) $(79.3 \%)$ and consequently high incidence of Respiratory Distress Syndrome (RDS). However, this rate is low if compared to $19.2 \%, 17.8 \%$ and $29 \%^{27,33,34}$ in other countries. This difference may be due to different patient 
characteristics like history of chorioamnionitis, late gestational age at

Table (4): Multivariate logistic regression analysis to identify predictors of poor fetal/neonatal outcome of PPROM.

\begin{tabular}{lllll} 
& & \multicolumn{3}{c}{$\mathbf{9 5 \%}$ CI } \\
\multicolumn{1}{c}{ Covariates } & Odds ratio & \multicolumn{2}{c}{ for Odds ratio } & \\
\cline { 3 - 4 } & & Lower & Upper & \\
\hline Employment & 1.9 & 1.2 & 3.5 & $\mathbf{0 . 0 2}$ \\
Type of PPROM & 1.9 & 1.4 & 3.6 & $\mathbf{0 . 0 3}$ \\
Chorioamnionitis & 14.5 & 12.4 & 132.1 & $\mathbf{0 . 0 1}$ \\
Mode of delivery & 2.5 & 2.01 & 4.5 & $\mathbf{0 . 0 0 *}$ \\
Later gestational age at PPROM & 0.8 & 0.21 & 0.90 & $\mathbf{0 . 0 0 *}$ \\
Gravidity & & 0.67 & & 0.67 \\
Multi gravidity & 0.7 & 0.38 & 1.4 & 0.38 \\
Grand gravidity & 0.8 & 0.77 & 2.1 & 0.77 \\
Multiple pregnancy & 0.7 & 0.38 & 1.5 & 0.38 \\
Chronic diseases & 1.1 & 0.60 & 2.2 & 0.60 \\
Smoking & 1.5 & 0.67 & 12.3 & 0.67 \\
Maternal age & & 0.144 & & 0.14 \\
- 21-30years & 1.3 & 0.46 & 2.8 & 0.46 \\
• 30years & 2.4 & 0.06 & 6.2 & 0.06 \\
Previous history of PPROM & 0.4 & 0.24 & 1.7 & 0.24 \\
\hline
\end{tabular}

* Statistically significant difference

PPROM \& rate of preterm delivery in previous studies.

In the current study, $47 \%$ of infants were admitted to NICU which is low as compared to $65.3 \%$ and $75.8 \%$ in other studies. ${ }^{32,35}$ The higher rate of admission compared to the current study was described as their studies were performed on preterm babies; therefore more problems and higher rate of NICU admission compared to present study that included infants born at a wider range of gestational ages.

However, lower rates of NICU admission in the current study may be attributed to high rate of referral to other hospitals. Ain Shams University suffers from relative shortage of places in NICU in relation to the high number of deliveries received daily by the hospital. Admission of the needed cases may pose a problem. In the current study $2 \%$ of infants had neonatal sepsis, similarly, Nabhan et al., 2014 reported that 3.5\% of infants of mothers having PPROM at Ain Shams maternity hospital developed sepsis. ${ }^{36}$ But this rate is low if compared to rates of $18 \%$ in a study in Assuit and 6.7\% in
Bangladesh [16 \& 31]. The higher rate of sepsis reported in these studies may be due to difference in diagnostic criteria as diagnosis of sepsis was based on histopathological findings rather than clinical diagnosis as performed in the current study.

Working mothers had more risk for having poor fetal outcome with Odds ratio 1.9 (95\% CI: 1.2 - 3.5). This may be caused by work related stress; exerting effort in work and difficult transportation in Egypt may induce PPROM with all its consequences. This result goes in agreement with Sathenahalli et al., 2016 who reported that maternal manual labor during pregnancy was associated with higher incidence of prematurity and low birth weight. ${ }^{37}$ The lack of significant association in the present study between smoking \& poor fetal outcome was similarly reported in Zambia. ${ }^{18}$

There was no significant association between previous history of PPROM \& poor fetal outcome. Lack of association is probably due to that past history of PPROM is mostly related to subsequent occurrence of PPROM rather than its poor or good outcome. 
CS delivery was associated with risk of poor fetal outcome (Odds=2.5). Similarly in Jordan, delivery by CS was identified as a risk factor for NICU admission which is considered as one of the poor fetal outcomes [38]. This association is explained as CS, usually, reflects worse condition either maternal; as oligohydramnios due to gush of liquor, or fetal causes; like fetal distress -that necessitates ICU admission- or even fetal death. Also, chorioamnionitis was a significant predictor of poor fetal outcome similar to a study conducted in China. ${ }^{39}$

The earlier gestational age at PPROM in the present study was significantly associated with poor fetal outcome which agrees with other studies. ${ }^{8,22,40}$ This is explained by the fact that incidence of respiratory distress syndrome and the length of hospital stay are related to gestational age

More efforts should be done to decrease the occurrence of PPROM through adequate antenatal care and health education of pregnant women about risk factors of PPROM. To improve neonatal outcome; efforts should be done to improve Ain Shams neonatal intensive care units (NICUs), supplying them with better equipment and increase their number so that they become suitable to deal with such kind of complications.

\section{Study Limitations:}

Secondary data collection has its known limitations such as lack of completeness or sometimes doubted accuracy. Incompleteness was avoided by conducting a pilot study that revealed the available data in patient files before conducting the study. However there were some important missing data due to lack in the medical files as maternal factors which-in previous studies- were found to be significantly associated with LBW, included mother's knowledge (eg., importance of antenatal care visits), her health status and family's income, antenatal care follow up, some maternal complications like (endometritis, wound infection and puerperal sepsis) \& fetal complication (cord prolapse, inter ventricular hemorrhage). These factors could not be studied in the current study and may have a relationship with LBW in Egyptian mothers if studied effectively.

\section{Acknowledgement:}

We would like to thank the statistical unit staff in Ain Shams maternity hospital for their cooperation and for facilitating this work.

\section{References:}

1. Modena $\mathrm{AB}$, Kaihura $\mathrm{C}$ and Fieni $\mathrm{S}$ (2004). Pre-labour rupture of the membranes: recent evidence. Acta Biomed; 75:5-10.

2. Hernández Y, López Farán JA and Gámez C (2011). Comparison of maternal and perinatal outcomes in the conservative treatment preterm premature membrane rupture between the use of Erythromycin and clindamycin. Ginecol Obstet Mex; 79(7):403-10.

3. Noor S, Nazar AF, Sultana $R$ and Bashir R (2007). Prevalence of PPROM and its outcome. J Ayub Med Coll Abbottabad; 19(4): 7-14.

4. Osaikhuwuomwan J (2010). Preterm Rupture of membranes: the vitamin $\mathrm{C}$ factor. AJOL; 12(1):60-8.

5. Mercer BM (2003). Preterm premature rupture of the membranes. Obstet Gynaecol; 101(1): 178-93.

6. Mercer BM (2012). Antibiotics in the management of PROM and preterm labor. Obstet Gynecol Clin North Am; 39: 65-76.

7. Dars S, Malik S and Samreen I (2014). Maternal morbidity and perinatal outcome in preterm premature rupture of membranes before 37 weeks gestation. Pak J Med Sci; 30(3): 626-29.

8. Patil S and Patil V (2014). Maternal and Fetal Outcome in Premature Rupture 
of Membranes. IOSR Journal of Dental and Medical Sciences; 13(12): 56-83.

9. Caughey AB, Robinson JN and Norwitz ER (2008). Contemporary diagnosis and management of preterm premature rupture of membranes. Rev Obstet Gynecol; 1:11-22.

10. AL-Riyami N, Al-Ruheili I, AlShezaw F and Al-Khabori M (2013). Extreme Preterm Premature Rupture of Membranes: Risk Factors and FetoMaternal outcomes. Oman Med J; 28(2): 108-11.

11. Tavassoli F, Ghasem M, Mohamadzade A. and Sharifian J (2010). Survey of Pregnancy Outcome in Preterm Premature Rupture of Membranes with Amniotic Fluid Index $<5$ and $\geq 5$. Oman Med J; 25(2): 118-23.

12. Buhimschi C, Norman JE, Creasy RK, Resnik R, Iams JD, Lockwood CJ, Moore TR and Greene MF (2013). Pathogenesis of spontaneous preterm labor maternal fetal medicine: principles and practice. 7 ed. Philadelphia: Saunders/Elsevier,; 599-623

13. Okeke TC, Enwereji JO, Okoro OS, Adiri CO, Ezugwu EC and Agu PU (2014). The Incidence and Management Outcome of Preterm Premature Rupture of Membranes (PPROM) in a Tertiary Hospital in Nigeria.; 2 (1): 14-17. American College of Obstetricians and Gynecologists, Committee on Practice Bulletins-Obstetrics (2007). Premature rupture of membranes. Clinical management guidelines for obstetriciangynecologists. Obstet Gynecol;109 (4):1007-19.

14. Linehan L, Walsh $\mathrm{J}$ and Morris A (2016). Neonatal and maternal outcomes following mid trimester preterm premature rupture of the membranes: a retrospective cohort study. BMC Pregnancy and Child birth BMC series; 16-25.

15. Mohamad HS (2013). The Management of Pregnancies Complicated by Late Preterm Pre-labour Rupture of Membranes between 34 and
37 Weeks of Gestation. Med. J. Cairo Univ; 81(1): 929-34.

16. Gahwagi M.M.M, Busarira M.O and Atia M (2015). Premature Rupture of Membranes Characteristics, Determinants, and Outcomes of in Benghazi, Libya. Open Journal of Obstetrics and Gynecology; 5, 494-504.

17. Silwimba W (2014). Epidemiology of preterm premature rupture of fetal membranes at the university teaching hospital. Lusaka; MS. Zambia.

18. Fishman SG and Gelber SE (2012). Evidence for the clinical management of chorioamnionitis. Semin Fetal \& Neonatal Medicine; 17:46-50.

19. Endale T, Fentahun $\mathrm{N}$ and Gemada $\mathrm{D}$ (2016). Maternal and fetal outcomes in term premature rupture of membrane. World J Emerg Med; 7(2):147-52.

20. Moustafa ME and Gadallah MAH (2012). Student notes on medical statistics and research methods. 4th edition. Inferences from samplesConfidence interval; (42-51).

21. Chakraborty $B$, Mandal $T$ and Chakraborty S (2013). Outcome of Prelabor Rupture of Membranes in a Tertiary Care Center in West Bengal. Indian Journal of Clinical Practice; 24 (7).

22. Afza NS, Nargis $\mathrm{W}$ and Ahmed BU (2011). The incidence, risk factors and common fetal outcome of chorioamnionitis in women with preterm premature rupture of membrane (PPROM): A single centre study. J Obstet Gynaecol; 26(1): 10-19.

23. Obi SN and Ozumba BC (2007). Pre-term premature rupture of fetal membranes: the dilemma of management in a developing nation. $\mathbf{J}$ Obstet Gynaecol; 27(1):37-40

24. Shukla P, Bhargava $M$ and Disha (2014). "Study of Maternal and Fetal Outcome in Preterm Premature Rupture of Membrane". Journal of Evolution of Medical and Dental Sciences; 3(07) 17:1789-15. 
25. American College of Obstetricians and Gynecologists Committee on Practice Bulletins-Obstetrics (2016). Premature rupture of membranes. Clinical management guidelines for obstetrician-gynecologists. Obstet Gynecol;127(1):39-51.

26. Tahir $\mathrm{S}$, Aleem $\mathrm{M}$ and Aziz $\mathrm{R}$ (2002). Incidence and outcome of preterm premature rupture of membranes. Pak J Med Sci; 18(1):26-32. 27. Azzaz MSE, Maestre MAM, Cardoso RT (2016). Antenatal care visits during pregnancy and their effect on maternal and fetal outcomes in preeclamptic patients. J Obstet Gynaecol Res; 42(9):1102-10.

28. EL-Zanaty, F and Way A (2016) . Egypt Demographic and Health Survey 2008. Cairo, Egypt: Ministry of Health, Available

from:http://www.measuredhs.com.

29. El-Messidi A and Cameron A (2010). Diagnosis of premature rupture of membranes: Inspiration from the past and insights for the future. J Obstet Gynaecol Can; 32:561-69.

30. Akter $S$, Akter $R$ and Rashid $M$ (2010). Preterm Prelabour Rupture of the Membrane \&Feto-Maternal outcome: an Observational Study. Journal of Bangladesh College of Physicians and Surgeons.; Vol 28, No 1.

31. Boskabadi H, Maamouri $G$ and Mafinejad S (2011). Neonatal Complications Related with Prolonged Rupture of Membranes. Macedonian Journal of Medical Science.; 4: 93-98

32. Yang LC, Donald RT, Kaufman, Hume R, Calhour B and Howard HK (2004). Maternal and Fetal Outcomes of Spontaneous Preterm Premature Rupture of Membranes. J Amer Osteopath Assoc; 104: 537-42.
33. Mehmet A, Osmanaðaoðlu and SevilayÜnal (2005). Chorioamnion it is risk and neonatal outcome in preterm premature rupture of membranes. Arch Gynecol Obstet; 271: 33-9.

34. Tanir HM, Sener T, Aksit A, Ardic $\mathrm{N}$ and Tekin N (2003). PROM and Neonatal Outcome prior to 34 weeks of Gestation. Int J GynaecolObstet;82(2): 16772.

35. Nabhan AF, Elhelaly A and Elkadi M (2014). Antibiotic prophylaxis in prelabor spontaneous rupture of fetal membranes at or beyond 36 weeks of pregnancy .Int $\mathrm{J}$ Gynaecol Obstet; 124(1):59-62.

36. Sathenahalli VB, Hussain Z, Gornale $\mathrm{V}$ and Singh HP (2016). Influence of maternal biosocial factors in fetal outcome. International Journal of Medical Science and Public Health.; 5(3).

37. Quinn CE, Sivasubramaniam

P, Blevins M, Al Hajajra A, Znait AT, Khuri-Bulos N, Faouri S and Halasa N (2016). Risk factors for neonatal intensive care unit admission in Amman, Jordan. East Mediterr Health J.; 22(3):163-74.

38. Zhang $\mathrm{H}$, Wang L, Wang J, Hei J, and Ruan C. (2015) Premature rupture of the fetal membrane combined with subclinical chorioamnionitis negatively affects pregnancy outcomes by a mechanism associated with reduced levels of matrix metalloproteinase-2. Exp Ther Med.; 10(2): 561-66.

39. Deutsch A, Deutsch E and Totten C. ,Maternal and neonatal outcomes based on the gestational age of mid-trimester preterm premature rupture of membranes. J Matern Fetal Neonatal Med. 2010; 23(12):1429-34. 